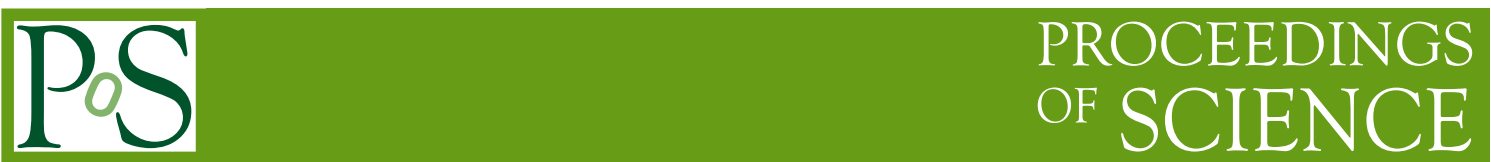

\title{
Massive Gravity with Two Stückelberg Fields
}

\section{Lasma Alberte*}

Ben-Gurion University, Beer Sheva, Israel

E-mail: lasmaepost.bgu.ac.il

\section{Andrey Khmelnitsky}

Ben-Gurion University, Beer Sheva, Israel

E-mail: andreykhepost.bgu.ac.il

\begin{abstract}
We consider the non-linear massive gravity as a theory of a number of Stückelberg scalar fields minimally coupled to the Einstein-Hilbert gravity and argue that the counting of degrees of freedom can be done for scalar theory and gravity separately. We investigate the system with only two Stückelberg scalar fields. In this case we find the analytic expression for the determinant of the kinetic matrix of the scalar field Lagrangian and perform the full constraint analysis. In $1+1$ space-time dimensions the theory corresponds to the full non-linear massive gravity, and this determinant vanishes identically. In this case we find two first-class constraints, and present the corresponding gauge symmetry of the theory which eliminates both scalar degrees of freedom. In $3+1$ dimensions the determinant of the kinetic matrix does not vanish identically and, for generic initial conditions, both scalar fields are propagating. We show how the massive gravity with two Stückelberg fields can be used in holographic context as a bulk description of a boundary field theory with momentum dissipation.
\end{abstract}

Frontiers of Fundamental Physics 14

15-18 July 2014

Aix Marseille University (AMU) Saint-Charles Campus, Marseille, France

\footnotetext{
*Speaker.
} 


\section{Introduction and Summary}

The theory of massive gravity necessarily invokes a reference metric, usually denoted by $f_{\mu \nu}$ [1], which as an absolute object breaks the diffeomorphism invariance of general relativity. It can be restored by introducing four Stückelberg scalar fields $\phi^{A}, A=0,1,2,3$ corresponding to the Goldstone bosons of the four broken coordinate transformations as [2, 3]:

$$
f_{\mu \nu}=\partial_{\mu} \phi^{A} \partial_{\nu} \phi^{B} f_{A B}(\phi),
$$

with $f_{A B}(\phi)-$ a configuration space reference metric. Then, a generic theory of massive gravity with the four Stückelberg scalars together with the two degrees of freedom of massless graviton propagates six degrees of freedom in total. It is one degree of freedom too much in comparison to the five degrees of freedom expected from the massive spin-2 representations of the Poincaré group. Moreover, the additional degree of freedom is sick and represents the Boulware-Deser ghost [1].

Contrary to the above expectations, the full resummed theory of the non-linear massive gravity of de Rham, Gabadadze, and Tolley (dRGT) [4] has been demonstrated to propagate only five degrees of freedom due to the special structure of the graviton mass term [5] (for a recent review, see [6]). In order to understand how this can be realised, it is useful to observe that the dRGT massive gravity in Stiuckelberg formulation is reparametrization invariant, and the scalars are coupled to gravity minimally. The degrees of freedom propagated by the metric and by the scalar fields can therefore be counted separately. Hence, the absence of the sixth mode in dRGT theory should manifest itself as the feature of the scalar fields Lagrangian alone. In other words, in dRGT theory the four Stückelbergs do not correspond to four independent degrees of freedom. This can be seen from the vanishing of the determinant of the kinetic matrix of the scalar field Lagrangian [7]

$$
\mathscr{A}_{A B} \equiv \frac{\partial^{2} \mathscr{L}_{\phi}}{\partial \dot{\phi}^{A} \partial \dot{\phi}^{B}} .
$$

Hence the equations of motion of the scalar fields are not independent from each other, and, as a result, in dRGT massive gravity the scalar fields propagate at most five degrees of freedom. However, up to our knowledge, the exact constraint structure of the scalar field part of the full dRGT massive gravity allowing to disentangle the actual degrees of freedom propagating in the scalar field sector is not known.

Motivated by these considerations we study the dynamics of the Stiuckelberg scalar fields given by the dRGT mass term. We observe that, if seen as a particular scalar field theory, the dRGT scalar field Lagrangian allows for an arbitrary number of scalar fields in it. The resulting theory can be regarded as a specific scalar-tensor theory with one or multiple scalar fields. In such setting, even some well-known scalar theories like k-essence, string theory, or the effective field theory of fluids, can be regarded as dRGT-type theories.

Here we present the results of [8] where we have considered the case of reduced massive gravity with two Stückelberg fields. It is the simplest case with several scalar fields involved, in which we can write the Hamiltonian and constraint structure explicitly. We perform the full Hamiltonian analysis of the scalar field sector and find that, in distinction from the dRGT massive gravity the determinant of the kinetic matrix does not vanish. Hence the scalar field Lagrangian in general propagates two degrees of freedom in this case. 
Recently, massive gravity with two Stückelberg fields has been successfully used in order to describe the transport properties in materials with broken translational invariance via the AdS/CFT correspondence [9]. When extended with a negative cosmological constant and a minimally coupled $U(1)$ gauge field this theory can be used as a gravity dual of a field theory with broken translational invariance. By considering this theory as the bulk gravitational theory on the black brane background, the calculated conductivity in the holographic framework is finite. This is different from the same calculation performed in the Einstein-Maxwell theory, where the diffeomorphism invariance in the bulk implies a translational symmetry in the field theory thus leading to infinite conductivity. Instead, in massive gravity the background solutions for Stükelberg fields break the space-time diffeomorphisms, which allows for momentum dissipation in the boundary theory and renders the conductivity finite [9] (see also [10, 11]). In [12] we have generalised the previous calculations to the diffeomorphism invariant case of massive gravity with two Stückelberg fields and performed the stability analysis of the perturbations around the black brane solution. Here we present the results of the calculations of the holographic conductivity.

\section{The kinetic matrix}

The dRGT massive gravity action is given by ${ }^{1}$

$$
\mathscr{L}_{E H}+m^{2} \mathscr{L}_{\phi}=\frac{M_{P}^{2}}{2} \sqrt{-g} R+m^{2} \sqrt{-g} \sum_{n=0}^{4} \beta_{n} \mathrm{e}_{n}(\sqrt{\mathscr{I}}),
$$

where $\mathscr{I}_{B}^{A} \equiv g^{\mu v} \partial_{\mu} \phi^{A} \partial_{\nu} \phi^{C} f_{B C}(\phi)$, and the matrix square root is defined as $\sqrt{\mathscr{I}}_{C}^{A} \sqrt{\mathscr{I}}_{B}^{C}=\mathscr{I}_{B}^{A}$. The auxiliary metric $f_{A B}(\phi)$ sets a global symmetry of the theory given by its isometry group and is used to move the scalar field space indices $A, B, \ldots \mathrm{e}_{n}(\mathbb{X})$ denote the characteristic polynomials of a given matrix, defined in terms of the traces of the matrix. The coefficients $\beta_{n}$ are arbitrary.

In what follows we treat the mass term Lagrangian $\mathscr{L}_{\phi}$ simply as a Lagrangian describing four Stiuckelberg scalar fields coupled to gravity. The motivation of restricting the number of scalar fields to the number of space-time dimension in the context of non-linear massive gravity is that around the background solution $g_{\mu v}=\eta_{\mu v}, \phi^{A}=x^{\mu} \delta_{\mu}^{A}$ the metric perturbations have a Lorentz invariant mass term of the Fierz-Pauli form at the quadratic level. However, if seen as a theory of scalar fields, the Lagrangian $\mathscr{L}_{\phi}$ describes just some particular theory of derivatively coupled scalar fields, and depends only on their first derivatives. This theory is diffeomorphism invariant and, in principle, the number of scalar fields can be chosen arbitrary.

Here we focus on the case of two scalar fields $\phi^{A}=\left\{\phi^{0}, \phi^{1}\right\}$ in $3+1$ dimensions as the simplest non-trivial case inspired by the dRGT massive gravity. For this setup the matrix $\mathscr{I}_{B}^{A}$ is a $2 \times 2$ matrix and the only non-vanishing characteristic polynomials of the square root matrix $\sqrt{\mathscr{I}}_{B}^{A}$ can be explicitly expressed in terms of the $\operatorname{Tr} \mathscr{I}$ and $\operatorname{det} \mathscr{I}$ as

$$
\begin{array}{ll}
\mathrm{e}_{0}(\sqrt{\mathscr{I}})=1, & \mathrm{e}_{1}(\sqrt{\mathscr{I}})=\operatorname{Tr} \sqrt{\mathscr{I}}=(\operatorname{Tr} \mathscr{I}+2 \sqrt{\operatorname{det} \mathscr{I}})^{1 / 2}, \\
\mathrm{e}_{2}(\sqrt{\mathscr{I}})=\operatorname{det} \sqrt{\mathscr{I}}=\sqrt{\operatorname{det} \mathscr{I}} .
\end{array}
$$

\footnotetext{
${ }^{1}$ This action is written in a slightly different, but equivalent from than in [4]. A detailed comparison is given in [8].
} 
Since the $\beta_{0}$ term does not affect the dynamics of the scalar fields, in what follows we set $\beta_{0}=0$. For a specific choice of the coefficients $\beta_{n}$ and for the scalar field metric taken to be the Minkowski metric, i.e. $f_{A B}=\eta_{A B}$, the full theory $\mathscr{L}=\mathscr{L}_{E H}+m^{2} \mathscr{L}_{\phi}$ has a solution $g^{\mu v}=\eta^{\mu v}, \phi^{A}=x^{\mu} \delta_{\mu}^{A}$. The quadratic action for the metric perturbations $h^{\mu v} \equiv g^{\mu v}-\eta^{\mu v}$ in the unitary gauge where $f_{\mu \nu}=\operatorname{diag}(+1,-1,0,0)$ coincides with a Lorentz-violating Fierz-Pauli-type mass term given as $h^{\mu v} h^{\alpha \beta}\left(f_{\mu v} f_{\alpha \beta}-f_{\mu \alpha} f_{v \beta}\right)$. Due to the degeneracy of the reference metric $f_{\mu v}$, the diffeomorphism invariance is broken only in the spacetime directions $\left\{x^{0}, x^{1}\right\}$.

In order to separate the time derivatives of the scalar fields while keeping the space-time metric arbitrary, we employ the ADM formalism. In terms of the ADM variables $N, N^{i}, \gamma^{i j}$ for the metric components the matrix $\mathscr{I}_{B}^{A}$ can be expressed as

$$
\mathscr{I}_{B}^{A}=\left(-D \phi^{A} D \phi^{C}+S^{A C}\right) f_{B C},
$$

where $D \equiv \frac{1}{N}\left(\partial_{0}-N^{i} \partial_{i}\right)$, and the matrix $S^{A C} \equiv \gamma^{i j} \partial_{i} \phi^{A} \partial_{j} \phi^{C}$ depends only on the spatial derivatives of the scalar fields. The determinant of the kinetic matrix (1.2) can then be found to be:

$$
\begin{aligned}
\operatorname{det} \mathscr{A}_{A B}=- & g \operatorname{det} f \frac{\operatorname{det} S}{(\operatorname{det} \mathscr{I})^{2}}\left(\frac { \beta _ { 1 } ^ { 2 } } { ( \operatorname { T r } \mathscr { I } + 2 \sqrt { \operatorname { d e t } \mathscr { I } } ) ^ { 2 } } \left[\sqrt{\operatorname{det} \mathscr{I} \mathscr{I}_{B}^{A}\left(3 S \delta_{A}^{B}-2 S_{A}^{B}\right)-}\right.\right. \\
& \left.-(\operatorname{Tr} \mathscr{I}+2 S) \mathscr{I}_{B}^{A}\left(S_{A}^{B}-S \delta_{A}^{B}\right)-2 S \operatorname{det} S\right]+ \\
& +\frac{\beta_{1} \beta_{2}}{(\operatorname{Tr} \mathscr{I}+2 \sqrt{\operatorname{det} \mathscr{I}})^{3 / 2}}[\sqrt{\operatorname{det} \mathscr{I}}(S \operatorname{Tr} \mathscr{I}+4 \operatorname{det} S)- \\
& \left.\left.-\left(2 S \mathscr{I}_{B}^{A}+S_{B}^{A} \operatorname{Tr} \mathscr{I}\right)\left(S_{A}^{B}-S \delta_{A}^{B}\right)-2 S \operatorname{det} S\right]+\beta_{2}^{2} \operatorname{det} S\right)
\end{aligned}
$$

This expression is valid for any choice of the scalar field metric $f_{A B}(\phi)$ as long as it does not involve the time derivatives of the scalar fields. The determinant depends on temporal and spatial derivatives of the scalar fields. In general it has a non-zero value which depends on the choice of initial conditions. The only special case when the determinant vanishes identically is if we are considering a two-dimensional space-time where the matrix $S^{A B}$ is a matrix of rank one, and $\operatorname{det} S \equiv 0$. This case corresponds to the two-dimensional massive gravity and our findings are in agreement with the previous work by de Rham et al. [7].

We thus conclude that in general the Lagrangian $\mathscr{L}_{\phi}$ in the case of two Stückelberg fields describes two independent dynamic fields. However, on the surface $\operatorname{det} S=0$ the Lagrangian equations of motion are degenerate. A detailed study on this matter is carried out in [8].

\section{1+1 dimensions: massive gravity}

It can be shown that the dynamics of the scalar fields in the subspace where $\operatorname{det} S=0$ is equivalent to the dynamics of scalar fields in the case of $1+1$ space-time dimensions [8]. We therefore discuss the $1+1$-dimensional case in detail here. For simplicity, we present the case of a flat Minkowski background metric $g_{\mu \nu}=\eta_{\mu v}$, when the scalar field Lagrangian can be written as

$$
\mathscr{L}_{\phi}=2 \sqrt{\dot{\psi}_{+}+\psi_{+}^{\prime}} \sqrt{\dot{\psi}_{-}-\psi_{-}^{\prime}},
$$


where $\psi_{ \pm} \equiv \phi^{0} \pm \phi^{1}$. The conjugated momenta to the fields $\psi_{ \pm}$can be shown to satisfy two firstclass constraints that form a closed algebra. This indicates that there is a gauge symmetry in our theory. Indeed, the Lagrangian (3.1) is invariant under the gauge transformation:

$$
\begin{aligned}
& \psi_{-} \mapsto \psi_{-}-\frac{1}{2}\left(\varepsilon^{\prime}+\dot{\varepsilon}\right) \frac{\dot{\psi}_{-}-\psi_{-}^{\prime}}{\dot{\psi}_{+}+\psi_{+}^{\prime}}, \\
& \psi_{+} \mapsto \psi_{+}+\frac{1}{2}\left(\varepsilon^{\prime}-\dot{\varepsilon}\right),
\end{aligned}
$$

where $\varepsilon$ is a gauge parameter. Since the above symmetry transformation involves both, the gauge parameter $\varepsilon$ and its time derivative, then the number of degrees of freedom in the theory is reduced by two - the total number of first class constraints. Hence, we conclude that in the $1+1$ dimensional massive gravity, as well as on the subspace $\operatorname{det} S=0$ of the $3+1$-dimensional theory there are no degrees of freedom propagated by the scalar field Lagrangian.

\section{Holographic conductivity}

For applications in holographic context, we consider massive gravity with two Stückelberg fields together with a negative cosmological constant and a $U(1)$ gauge field:

$$
\mathscr{L}=\frac{1}{2} \sqrt{-g}\left[(R+2 \Lambda)-\frac{1}{4} F_{\mu v} F^{\mu v}+\beta_{1} e_{1}(\sqrt{\mathscr{I}})+2 \beta_{2} e_{2}(\sqrt{\mathscr{I}})\right],
$$

and choose the two Stückelberg fields $\phi^{A}$ in the transverse directions with $A=\{x, y\}$ and a reference metric $f_{A B}=\delta_{A B}=\operatorname{diag}(1,1)$. The equations of motion of the Stuickelberg scalar fields and the Maxwell field admit the background solutions $\phi^{A}=x^{\mu} \delta_{\mu}^{A}$ and $A_{t}=\mu\left(1-r / r_{h}\right)$. The Einstein equations admit the black brane solution for the background metric:

$$
d s^{2}=\frac{d r^{2}}{f(r) r^{2}}+\frac{-f(r) d t^{2}+d x^{2}+d y^{2}}{r^{2}}
$$

with the emblackening factor $f(r)$ determined in terms of the parameters $\beta_{1}, \beta_{2}, \Lambda, r_{h}$.

The conductivity in AdS/CFT correspondence is calculated in the framework of the linear response theory. For a detailed reference on the calculation of the conductivity in the holographic framework see [13]. Here we shall present only the basic formulae needed for the calculations. The full analysis can be found in [12]. The conductivity that relates the current density in a material to the electric field is determined in terms of the Maxwell field by the Kubo formula

$$
\sigma(\omega)=\left.\frac{1}{i \omega} \frac{A^{\prime}(r)}{A(r)}\right|_{r \rightarrow 0}-\frac{\beta_{1}}{8 \pi T}
$$

where $T$ denotes the temperature of the black brane and the function $A(r)$ is related to the transverse Maxwell field $A_{i}$ with $i=x$ or $y$. The superscript $r \rightarrow 0$ in the equation for the conductivity indicates that the function $A(r)$ is evaluated at the AdS boundary $r=0$. The numerical results of [12] for the real and imaginary parts of the conductivity for a particular set of parameters are shown in figure 1. We see that the real part of the conductivity exhibits a peak at zero frequency, and the imaginary part of the conductivity is finite. For comparison, in Einstein-Maxwell theory, the 

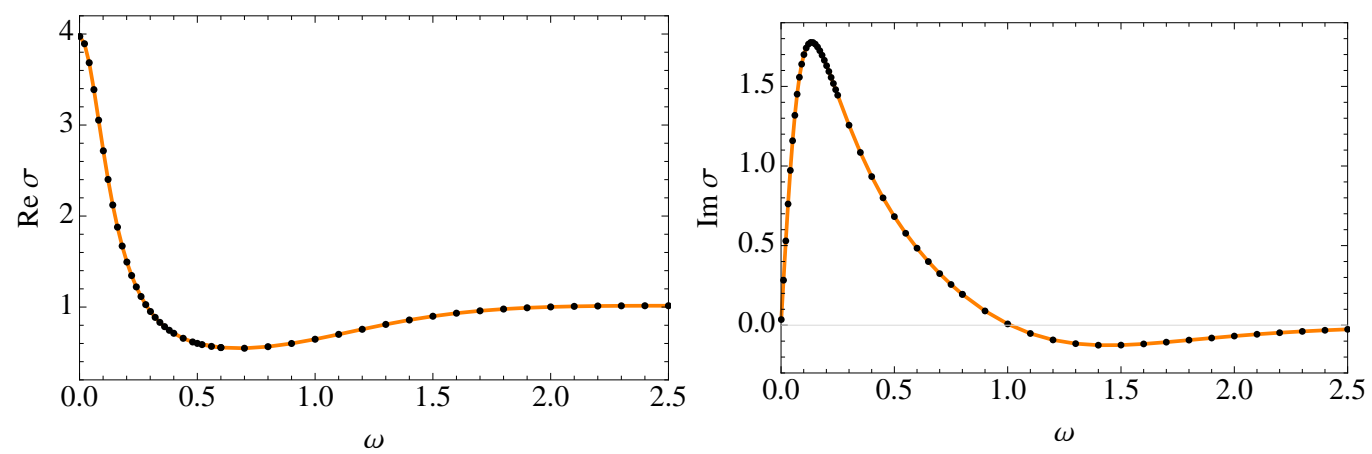

Figure 1: The real and imaginary part of the electrical conductivity. The parameters are set to $\beta_{1}=-1, \beta_{2}=0, \mu=1.724$.

imaginary part of the conductivity has a pole at $\omega=0$ and thus the real part of the conductivity is a delta function at $\omega=0$. This is a standard AdS/CFT result and is a well-known mismatch between the AdS/CFT predictions and the properties of real materials [13]. Hence, considering the reduced massive gravity as the gravitational theory in the bulk provides a framework for describing the momentum dissipation in the boundary field theory.

\section{References}

[1] D. G. Boulware and S. Deser, "Can gravitation have a finite range?,” Phys. Rev. D 6 (1972) 3368

[2] N. Arkani-Hamed, H. Georgi and M. D. Schwartz, "Effective field theory for massive gravitons and gravity in theory space," Annals Phys. 305 (2003) 96 [hep-th/0210184].

[3] A. H. Chamseddine and V. Mukhanov, "Higgs for Graviton: Simple and Elegant Solution," JHEP 1008 (2010) 011 [arXiv:1002.3877 [hep-th]].

[4] C. de Rham, G. Gabadadze and A. J. Tolley, "Resummation of Massive Gravity," Phys. Rev. Lett. 106 (2011) 231101 [arXiv:1011.1232 [hep-th]].

[5] S. F. Hassan and R. A. Rosen, "Resolving the Ghost Problem in non-Linear Massive Gravity," Phys. Rev. Lett. 108 (2012) 041101 [arXiv:1106.3344 [hep-th]].

[6] C. de Rham, "Massive Gravity," Living Rev. Rel. 17 (2014) 7 [arXiv:1401.4173 [hep-th]].

[7] C. de Rham, G. Gabadadze and A. J. Tolley, "Ghost free Massive Gravity in the Stückelberg language," Phys. Lett. B 711 (2012) 190 [arXiv:1107.3820 [hep-th]].

[8] L. Alberte and A. Khmelnitsky, "Reduced massive gravity with two Stückelberg fields," Phys. Rev. D 88 (2013) 6, 064053 [arXiv:1303.4958 [hep-th]]

[9] D. Vegh, "Holography without translational symmetry," arXiv:1301.0537 [hep-th].

[10] R. A. Davison, "Momentum relaxation in holographic massive gravity," Phys. Rev. D 88 (2013) 086003 [arXiv:1306.5792 [hep-th]].

[11] M. Blake and D. Tong, "Universal Resistivity from Holographic Massive Gravity," Phys. Rev. D 88 (2013) 106004 [arXiv:1308.4970 [hep-th]].

[12] L. Alberte and A. Khmelnitsky, "Stability of Massive Gravity Solutions for Holographic Conductivity," arXiv:1411.3027 [hep-th]

[13] S. A. Hartnoll, "Lectures on holographic methods for condensed matter physics," Class. Quant. Grav. 26 (2009) 224002 [arXiv:0903.3246 [hep-th]]. 